\title{
The role of angiotensin-(1-7) on acquired platinum resistance-induced angiogenesis in non-small cell lung cancer in vitro and in vivo
}

\author{
Yan-Lai GENG ${ }^{1,2,3, *}$, Yong-Jie DING ${ }^{1,2, *}$, Lei NI ${ }^{1,2}$, Kan-Di XU ${ }^{1,2}$, Van-Minh LE ${ }^{4}$, Ri JI ${ }^{5, *}$, Yun FENG ${ }^{1,2, *}$ \\ ${ }^{1}$ Department of Respiratory and Critical Care Medicine, Ruijin Hospital, Shanghai Jiao Tong University School of Medicine, Shanghai, China; \\ ${ }^{2}$ Institute of Respiratory Diseases, Shanghai Jiaotong University School of Medicine, Shanghai, China; ${ }^{3}$ Department of Respiration, Wuxi Branch \\ of Ruijin Hospital, Shanghai Jiao Tong University School of Medicine, Wuxi, China; ${ }^{4}$ Research Center of Ginseng and Medicinal Materials, Na- \\ tional Institute of Medicinal Materials, Ho Chi Minh City, Vietnam; ${ }^{5}$ Department of Ultrasound, Ruijin Hospital, Shanghai Jiaotong University \\ School of Medicine, Shanghai, China
}

*Correspondence: jiri_1980@163.com; fy01057@163.com

${ }^{*}$ Contributed equally to this work.

Received December 13, 2020 / Accepted March 23, 2021

\begin{abstract}
Renin-angiotensin system (RAS) signaling has been implicated in the development of cancer. The new RAS ACE2/ Ang-(1-7)/Mas axis antagonizes the classical ACE/Ang II/AT1R axis. Ang-(1-7) has pleiotropic roles in lung cancer including suppressing proliferation, angiogenesis, and metastasis. This research was designed to investigate the effect of Ang-(1-7) on tumor-associated angiogenesis in DDP-resistant lung cancer cell lines. We first established acquired DDP-resistant cell lines A549 (A549-DDP) and LLC (LLC-DDP). We next performed RT-qPCR, western blot, ELISA, tube formation, microvessel density detection, immunohistochemistry, and tumor formation assays. The results showed that the mRNA and protein levels of RAS components and vascular endothelial growth factor A (VEGFa) were lessened in the A549/ LLC-DDP+Ang-(1-7) group compared with the A549/LLC-DDP group. This effect could be blocked by the MAS receptor antagonist A779. The data revealed that Ang-(1-7) could perform its antiangiogenic function by PI3K/AKT and MAPK pathways. Furthermore, the impact of Ang-(1-7) on tumor-associated angiogenesis has been confirmed in lung cancer xenograft model with acquired DDP resistance. These results provide a theoretical basis for designing therapeutic strategies for targeting Ang-(1-7) in the treatment of NSCLC.
\end{abstract}

Key words: NSCLC, Ang-(1-7), acquired platinum resistance, tumor-associated angiogenesis, ACE2/Ang-(1-7)/Mas axis

Platinum-based drugs have a wide range of applications in the treatment of human neoplasms. However, the severe side effects and the rise of chemotherapy resistance have costed platinum drugs' clinical applications in many scenarios [1]. Moreover, there is plenty of evidence that tumor-associated angiogenesis is one of the mechanisms of tumor growth and metastasis led by acquired platinum resistance $[2,3]$.

The conventional renin-angiotensin system (RAS) contains renin, ACE, angiotensinogen, Angiotensin II (Ang II), Ang II type 1 subtype receptor (AT1R), and Ang II type 2 subtype receptor (AT2R). Angiotensin-(1-7) [Ang-(1-7)] is an important component of RAS ACE2/Ang-(1-7)/Mas axis. Ang-(1-7) can be generated in two ways: one originates from angiotensin I by endopeptidases and the other from angiotensin II cleaved by ACE2. Ang II acts as a vital effector in the classical RAS ACE/Ang II/AT1R axis. The new ACE2/
Ang-(1-7)/Mas axis functions as an antagonist against the conventional ACE/Ang II/AT1R axis $[4,5]$.

Previously, our team has proved that the ACE/Ang II/ AT1R axis participated in lung cancer development, which could be attenuated by ACEI and ARB [6, 7]. We found that ACE2 overexpression could reduce NSCLC tumor angiogenesis and invasion both in vitro and in vivo $[8,9]$. It was reported that VEGF expression is elevated in platinumresistant bladder cancer cells than in the parental cancer cells. Tumor acquired platinum resistance can regulate tumor angiogenesis by modulating AT1R [2]. We found that the ACE2 overexpression could inhibit acquired platinumresistant NSCLC angiogenesis [10]. Ang-(1-7) suppressed A549 cells metastasis via inhibition of MMP2 and MMP9 [11]. Here we intended to explore whether Ang-(1-7) could inhibit tumor angiogenesis in platinum-resistant NSCLC 
and understand the underneath molecular mechanisms in vitro and in vivo.

\section{Materials and methods}

Cell culture and stable cell line. A549 and LLC were cultured in Ham's F12 medium (Gibco BRL, Grand Island, NY) with $10 \%$ fetal calf serum (FCS), $100 \mathrm{mg} / \mathrm{ml}$ penicillin, and $100 \mathrm{U} / \mathrm{ml}$ streptomycin. Platinum resistance was achieved by culturing A549 and LLC in 10\% fetal bovine serum-containing CDDP. A549 and LLC cells were cultured in a medium with $3 \mu \mathrm{M}$ CDDP for 6 months [10]. Thus, we established platinum-resistant NSCLC cell lines named A549-DDP and LLC-DDP. A549, LLC, A549-DDP, and LLC-DDP cells were treated with various CDDP concentrations for $48 \mathrm{~h}$. The $\mathrm{IC}_{50}$ values for A549 and A549-DDP cells were $10.8 \pm 0.5$ and $39.6 \pm 1.8 \mu \mathrm{mol} / \mathrm{l}$, respectively. The resistance factor $(\mathrm{RF})$ was 3.67. The $\mathrm{IC}_{50}$ values for $\mathrm{LLC}$ and LLC-DDP cells were $8.5 \pm 0.4$ and $23.3 \pm 2.1 \mu \mathrm{mol} / \mathrm{l}$, respectively. The RF for these cells was 2.74. Ang-(1-7) and Ang-(1-7) receptor antagonist A779 were obtained from Bachem (Shanghai Institute of Cells). In the in vitro assays, A549/LLC-DDP cells were incubated with $10 \mathrm{nmol} / \mathrm{l}$ Ang-(1-7) in the group of A549/LLC-DDP+Ang-(1-7) for $24 \mathrm{~h}$ and $10 \mathrm{nmol} / \mathrm{l} \mathrm{Ang-(1-7)}$ in the presence of $100 \mathrm{nmol} / \mathrm{l}$ A779 in the group of A549/LLC-DDP+Ang-(1-7)+A779 for $24 \mathrm{~h}$. Prior to treatment, all cells were placed in a serumdepleted medium for $24 \mathrm{~h}$ to maintain quiescence. Transfection of cells was implemented with Lipofectamine ${ }^{\mathrm{Ts}} 2000$ (Thermo Fisher Scientific, 11668027, USA) according to the manufacturer's protocol. The si-Ang-(1-7) were synthesized by Hanbio Biotechnology Co., Ltd. (Shanghai, China). The siRNA sequence is 5'-CAGCAGUUCGGAAUGGCGCtt-3'.

Tumor growth assays. Six to eight-week-old BALB/c athymic nude mice were purchased from the Chinese Academy of Sciences, Shanghai. The animal protocol in this work was in accordance with the guideline for Laboratory Animal Ethics of Shanghai Jiao Tong University and followed the regulations for the Administration of Affairs Concerning Experimental Animals (China, 2014) and the National Institutes of Health Guide for Care and Use of Laboratory Animals (GB14925-2010). The mice were divided into the following 3 groups: 1) A549-DDP, 2) A549-DDP+Ang-(1-7), 3) A549-DDP+Ang-(1-7)+A779. Each mouse was inoculated with $1 \times 10^{6}$ A549 cells in the flank $(n=6)$. In the A549-DDP+Ang-(1-7) group, the animals received s.c. injections of $1,000 \mu \mathrm{g} / \mathrm{kg} / \mathrm{d}$ Ang-(1-7) $(\mathrm{n}=6)$ for 5 times/week. In A549-DDP+Ang-(1-7)+A779 group, each mouse received s.c. injections of $1,000 \mu \mathrm{g} / \mathrm{kg} / \mathrm{d}$ Ang-(1-7) [12] and was fed with $1,000 \mu \mathrm{g} / \mathrm{kg} / \mathrm{d}$ A779 for 5 times/week. Tumor growth was monitored every 3 days. After the next 33 days, the mice were euthanized and the tumors were removed.

qRT-PCR assay. Total RNA extraction was achieved by the Trizol reagent (Invitrogen). cDNA was synthesized with TransScript First-Strand cDNA Synthesis SuperMix (Trans- gene Biotech, Beijing, China) according to the instruction. Then, quantitative real-time polymerase chain reaction mixture was prepared (qPCR mixture: $10 \mu \mathrm{l}$ TransStart Top Green qPCR SuperMix (Transgene Biotech, Beijing, China); $4 \mu \mathrm{l}$ template $\mathrm{cDNA} ; 0.15 \mu \mathrm{l}$ forward primer $(50 \mu \mathrm{M}) ; 0.15 \mu \mathrm{l}$ reverse primer $\left.(50 \mu \mathrm{M}) ; 5.7 \mu \mathrm{ldd} \mathrm{H}_{2} \mathrm{O}\right)$. The sequences of primers are listed in Table 1.

Western blot analysis. RAS components and VEGFa levels were investigated in corresponding cells. Proteins were extracted and separated on a $10 \%$ SDS-PAGE gel ahead of being transferred on PVDF membranes (Life Technologies, USA). Then membranes were blocked in 5\% BSA-TBST for $1.5 \mathrm{~h}$ and probed with different primary antibodies overnight such as VEGFa and AT1R (Santa Cruz Biotechnology), ACE, and ACE2 (R\&D Systems). PI3K, p-PI3K, Akt, p-Akt, P38, p-P38, JNK, p-JNK, ERK1/2, and p-ERK1/2 antibodies were purchased from Cell Signaling Technology (Beverly, MA, USA). $\beta$-actin (Sigma Corporation) was used as a loading control.

Tube formation assay. Matrigel was diluted in Ham's F12 medium without serum (medium: Matrigel $=2: 1$ ). $30 \mu \mathrm{l}$ of diluted Matrigel was added to each well of a 96well plate and allowed to solidify at $37^{\circ} \mathrm{C}$ for $0.5 \mathrm{~h}$. A549/ LLC, A549/LLC-DDP, and A549/LLC- DDP supplemented with Ang-(1-7) or Ang-(177)+A779 were grown for $48 \mathrm{~h}$ in FCS-free media prior to incubation with different fatty acids in the presence of 5\% FCS media. Cells were seeded at a density of 50,000 cells/well. After 24 h culture, 8 randomly selected images were acquired under a microscope. The experiment was repeated three times.

Immunohistochemical staining. Formalin-fixed and paraffin-embedded sections were dissected from the tissues at a thickness of $5 \mu \mathrm{m}$. Sections were deparaffinized in xylene and rehydrated with graded ethanol to remove water. Antigen retrieval was not performed. Then sections were blocked with $1 \%$ bovine serum albumin (Invitrogen) in phosphatebuffered saline (PBS) for 30 minutes. The sections were incubated with antibody against Ki-67 (1:50, clone MIB-1; Dako), anti-human antibody against VEGFa (1:200, Santa Cruz Biotechnology, Santa Cruz, CA, sc-152) overnight at $4{ }^{\circ} \mathrm{C}$. The sections were incubated at room temperature with the secondary antibody (1:200, Santa Cruz Biotechnology, Santa Cruz, CA, sc-2357) for 20 min. Diaminobenzidine (DAB) staining was then performed. The scoring of the NSCLC sample was carried out by a histopathologist by calculating at least 500 tumor cells in five different fields of view for each specimen.

Table 1. qRT-PCR primer sequences.

\begin{tabular}{ll}
\hline Gene & Primers \\
\hline ACE & F:5'-CCGATCTGGCAGAACTTC-3' \\
& R:5'-GTGTTCCAGATCGTCCTC-3' \\
ACE2 & F:5'-CCACTGCTCAACTACTT TGAGCC-3' \\
& R:5'-CTTATCCTCACTTTG ATGCT-3' \\
\hline
\end{tabular}


Microvessel density detection. MVD was performed as conducted by Weidner et al. and Maeda et al. CD31 antibody (Dako, Glostrup, Denmark) was incubated overnight at $4{ }^{\circ} \mathrm{C}$, followed by peroxidase-labeled biotinylated anti-rat antibody at RT for $30 \mathrm{~min}$. Then the sections were incubated in streptavidin-HRP (1:300) for $30 \mathrm{~min}$, then the DAB substrate and counterstained with hematoxylin. Brown-yellow stained endothelial cells or cell plexuses were considered as a blood vessel. Within the hotspot region, microvessels were counted under $200 \times$ magnification, and the average of vessel counts in three hotspots was considered as MVD value. All counts were performed randomly by three investigators.

ELISA assay. The cells were serum-starved for $24 \mathrm{~h}$. The supernatant was collected and VEGFa levels were measured by VEGFa ELISA kit (R\&D Systems, Minneapolis, MN) according to the manufacturer's instructions.

Statistical analysis. Statistical analysis was performed using GraphPad Prism 5.0. We usually presented the results as mean \pm SD of biological replicates. Student's t-test was used to evaluate the difference between each test group and the control group.

Ethics guidelines. All institutional and national guidelines for the care and use of laboratory animals were followed.

\section{Results}

Role of Ang-(1-7) on VEGFa production and RAS elements in vitro. We explored whether Ang-(1-7) inhibited the VEGFa production in A549-DDP and LLC-DDP. Western blot analysis showed that the protein levels of ACE, AT1R, and VEGFa increased and the protein level of ACE2 decreased in the A549-DDP group compared to those in the A549 group, while the protein expressions of ACE, AT1R, and VEGFa declined in the A549-DDP+Ang-(1-7) group compared with the A549-DDP group. The MAS receptor antagonist A779 could inhibit this effect (Figure 1A). We also performed an ELISA assay to detect the VEGFa productions in supernatants. The result was consistent with the western blot, as shown in Figure 1B. We applied the same methods to study the impact of Ang-(1-7) on VEGFa production and RAS components in LLC-DDP cell lines and the consequence was consistent with A549-DDP (Figures 1C, 1D). A549/LLC-DDP significantly increased capillary network complexities and induced more tubes in the network compared with parental cells. A549/ LLC-DDP treated with Ang-(1-7) showed a decreased tube length compared to the A549/LLC-DDP, which could be reversed by A779 (Figures 2A-2D).
A

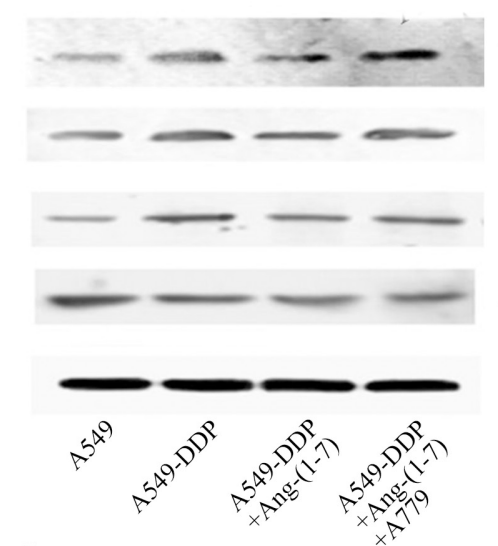

$\mathrm{C}$

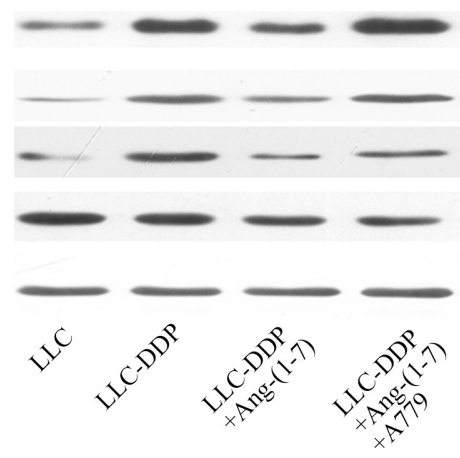

B

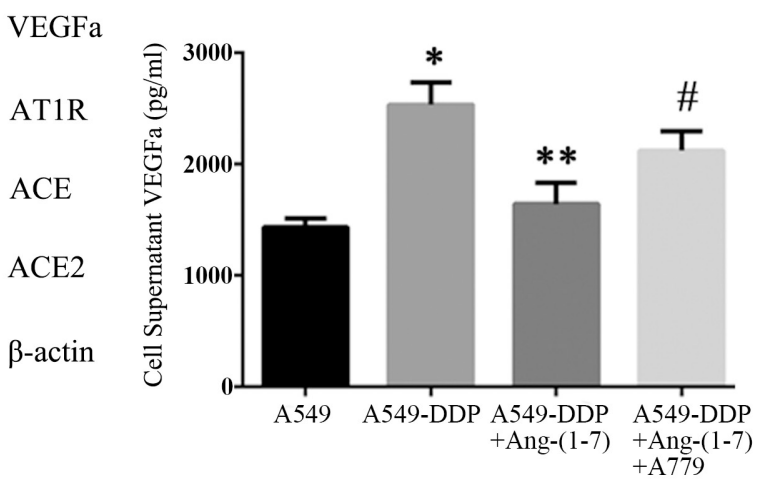

D

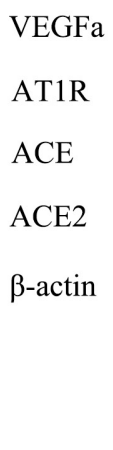

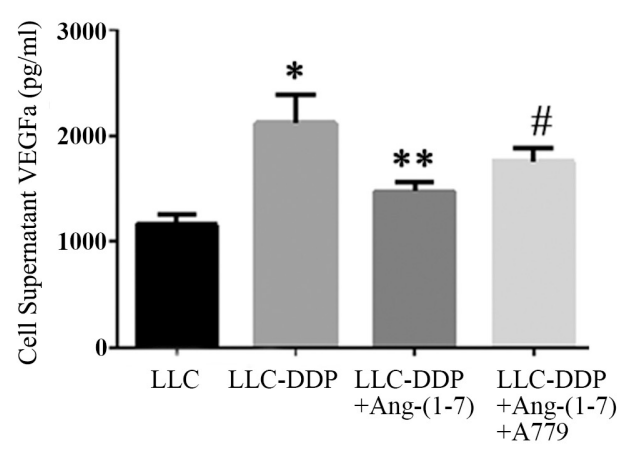

Figure 1. The effects of Ang-(1-7) on VEGFa production and RAS components in vitro. A) The effects of Ang-(1-7) alone and a combination of Ang(1-7) and A779 on the production of ACE, VEGFa, ACE2, and AT1R in A549-DDP+Ang-(1-7) cells and A549-DDP cells. B) VEGFa protein levels in the A549 supernatants were determined by ELISA. C) The effects of Ang-(1-7) alone and a combination of Ang-(1-7) and A779 on the production of ACE, VEGFa, ACE2, and AT1R in LLC-DDP+Ang-(1-7) cells and LLC-DDP cells. D) VEGFa protein levels in the LLC supernatants were determined by ELISA (mean \pm SD) ${ }^{*}$ p $<0.05$ vs. A549, ${ }^{* *}$ p $<0.05$ vs. A549-DDP, \# $p<0.05$ vs. A549-DDP+Ang-(1-7). 
A

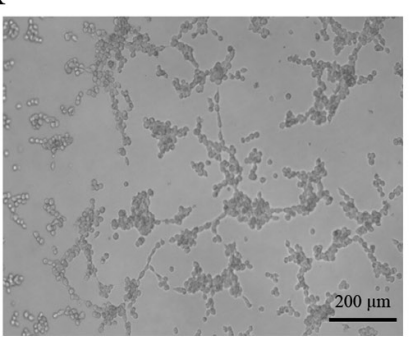

A549

B

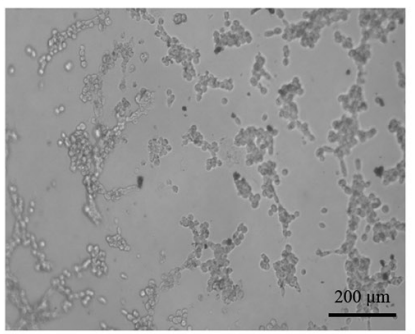

LLC

C

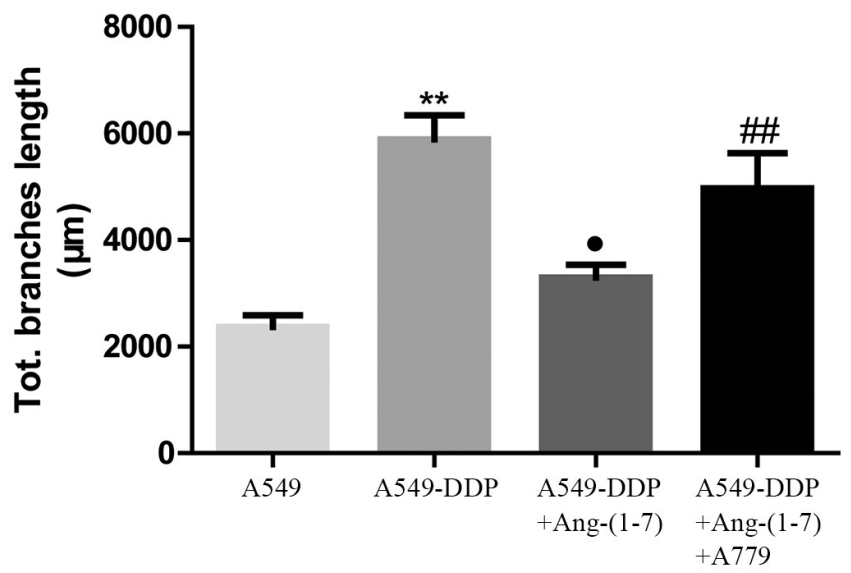

A549-DDP

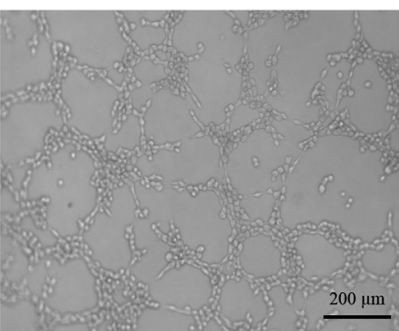

LLC-DDP

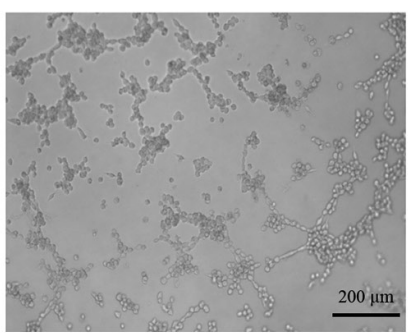

A549-DDP + Ang-(1-7)

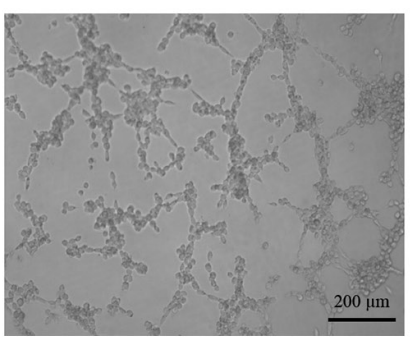

A549-DDP+Ang-(1-7)+A779

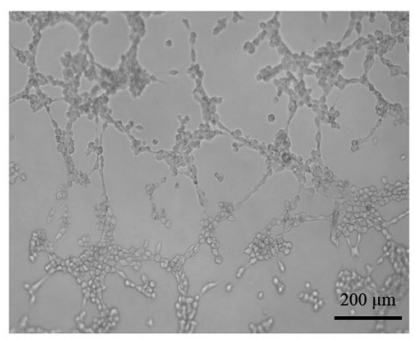

LLC-DDP+Ang-(1-7)

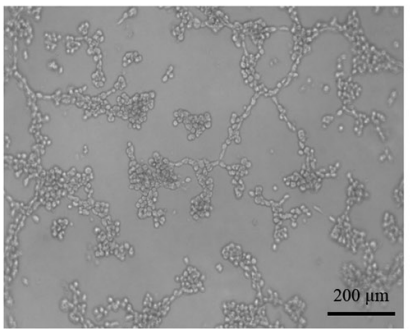

LLC-DDP+Ang-(1-7)+A779

$\mathrm{D}$

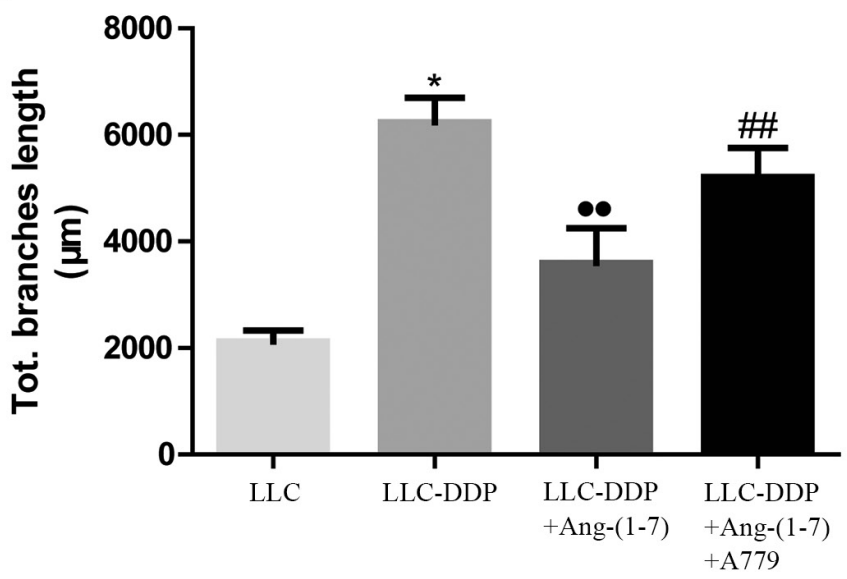

Figure 2. The effects of Ang-(1-7) on tube formation in the A549/LLC-DDP cells. A, B) Representative images of tube formation in the A549/LLC and A549/LLC-DDP cells cultured on Matrigel for $24 \mathrm{~h}$. C, D) The histograms indicate the relative length of tubes. Significant differences were indicated by ${ }^{\star} \mathrm{p}<0.05,{ }^{* *} \mathrm{p}<0.01$ vs. A549/LLC; ${ }^{\bullet p}<0.05,{ }^{*} \mathrm{p}<0.01$ vs. A549/LLC-DDP; ${ }^{\# \#} \mathrm{p}<0.01$ vs. A549/LLC-DDP+Ang-(1-7).

Transfection of exogenous small interfering RNA (siRNA) was used to demonstrate the function of Ang-(17). We synthesized si-Ang-(1-7) to inhibit Ang-(1-7)'s function and found that A549/LLC-DDP treated with si-Ang-(1-7) showed increased VEGFa productions and tube lengths compared to the A549/LLC-DDP (Supplementary Figures S1A-S1D).

The effect of Ang-(1-7) on VEGFa, ACE, ACE2, and AT1R mRNA levels. qPCR results of VEGF and RAS components mRNA levels demonstrated that the A549-DDP group has significantly higher levels of VEGFa, AT1R, and ACE mRNA (Figures 3A-3C), and lower mRNA expression of ACE2 (Figure 3D) compared with the A549 group. The ACE, AT1R, and VEGF mRNA expression decreased in the A549-DDP+Ang-(1-7) group than those in the A549-DDP group. We also found that their levels increased in the A549-DDP+Ang-(1-7)+A779 group compared with the A549-DDP+Ang-(1-7) group, while ACE2 expression did not change. The same results also were observed in LLC and LLC-DDP cells. 

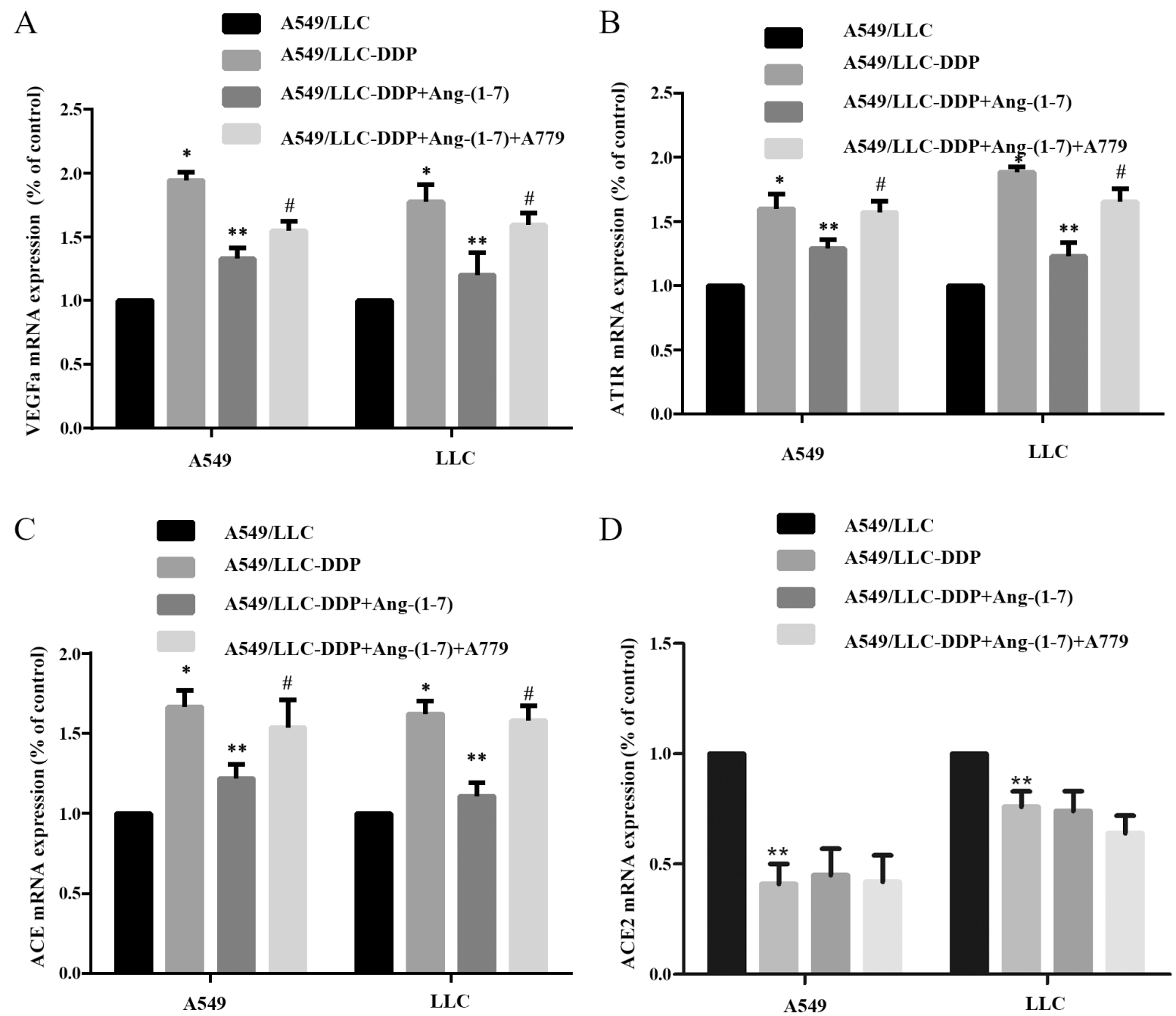

Figure 3. The effect of Ang-(1-7) on VEGFa mRNA production and RAS components in vitro. The effects of Ang-(1-7) on the mRNA levels of A) VEGFa, B) AT1R, C) ACE, and D) ACE2 in A549/LLC-DDP+Ang-(1-7) cells and A549/LLC-DDP cells (mean \pm SD). ${ }^{*}$ p $<0.05$ vs. A549/LLC, ${ }^{* *}$ p $<0.05$ vs. A549/ LLC-DDP, ${ }^{*} p<0.05$ vs. A549/LLC-DDP+Ang-(1-7)

Ang-(1-7) signaling is involved in the acquired DDP resistance-induced NSCLC angiogenesis. In order to understand the anti-angiogenic mechanism of Ang-(1-7) in the acquired platinum-resistant NSCLC cell lines, we conducted the western blot to investigate whether Ang-(1-7) has an effect on the PI3K/Akt and MAPK signaling (Figure 4A). To compare with the A549 group, the phosphorylation levels of PI3K/Akt, P38, and ERK1/2 signaling increased in the A549-DDP group, which was attenuated by Ang-(1-7). We also found that the phosphorylation levels of this signaling pathway increased in the A549-DDP+Ang-(1-7)+A779 group than those in the A549-DDP+Ang-(1-7) group (Figures 4B, 4C). Besides, the combination of Ang-(1-7) and the inhibitors of the PI3K/Akt and MAPK signaling could attenuate the expression of VEGF (Supplementary Figures S2A, S2B).
The data revealed that PI3K/Akt, P38, and ERK1/2 signaling inactivation have impacted the role of Ang-(1-7) on tumor angiogenesis induced by DDP resistance.

Tumor growth was prohibited by Ang-(1-7) in the acquired DDP-resistant lung cancer xenograft model. To explore the influence of Ang-(1-7) on tumor growth, we divided the mice into three groups, A549-DDP, A549-DDP+Ang-(1-7), and A549-DDP+Ang-(1-7)+A779. As shown in Figures $5 \mathrm{~A}$ and $5 \mathrm{~B}$, in comparison with the A549-DDP group, the tumor volume and weight decreased in the A549-DDP+Ang-(1-7) group and increased in the A549-DDP+Ang-(1-7)+A779 group. The consequence of tumor growth curves is consistent with tumor weight (Figure 5C). The proliferation-related gene Ki-67 was measured by immunohistochemistry. Ki-67 staining inten- 
sity was weaker in the A549-DDP+Ang-(1-7) group than that in the A549-DDP group (Figure 5D). The results showed that Ang-(1-7) could prohibit tumor growth in the acquired DDP-resistant lung cancer xenograft model.

Ang-(1-7) inhibited the VEGFa production in the acquired DDP-resistant lung cancer xenograft model. We next explored the role of Ang-(1-7) in the VEGFa expression in the acquired DDP-resistant lung cancer xenograft model. We found that the A549-DDP+Ang-(1-7) group showed significantly lower expression levels of ACE, AT1R, and VEGFa than that of the A549-DDP group (Figure 6A). IHC staining also showed that Ang-(1-7) prohibited the VEGFa expression (Figure 6B). We hypothesized that Ang-(1-7) influenced angiogenesis in the acquired DDP-resistant lung cancer xenograft model. Therefore, we further explored microvessel density (MVD). To compare with the A549-DDP group, endothelial-specific marker CD34-positive staining was lower in the A549-DDP+Ang-(1-7) group. MVD of the A549-DDP+Ang-(1-7)+A779 group was significantly higher than that of the A549-DDP+Ang-(1-7) group (Figures 6C, 6D). The results in vivo demonstrated that RAS components and VEGFa expression were lower in the A549-DDP+Ang-(1-7) group than those of the A549-DDP group, which was consistent with the in vitro results.

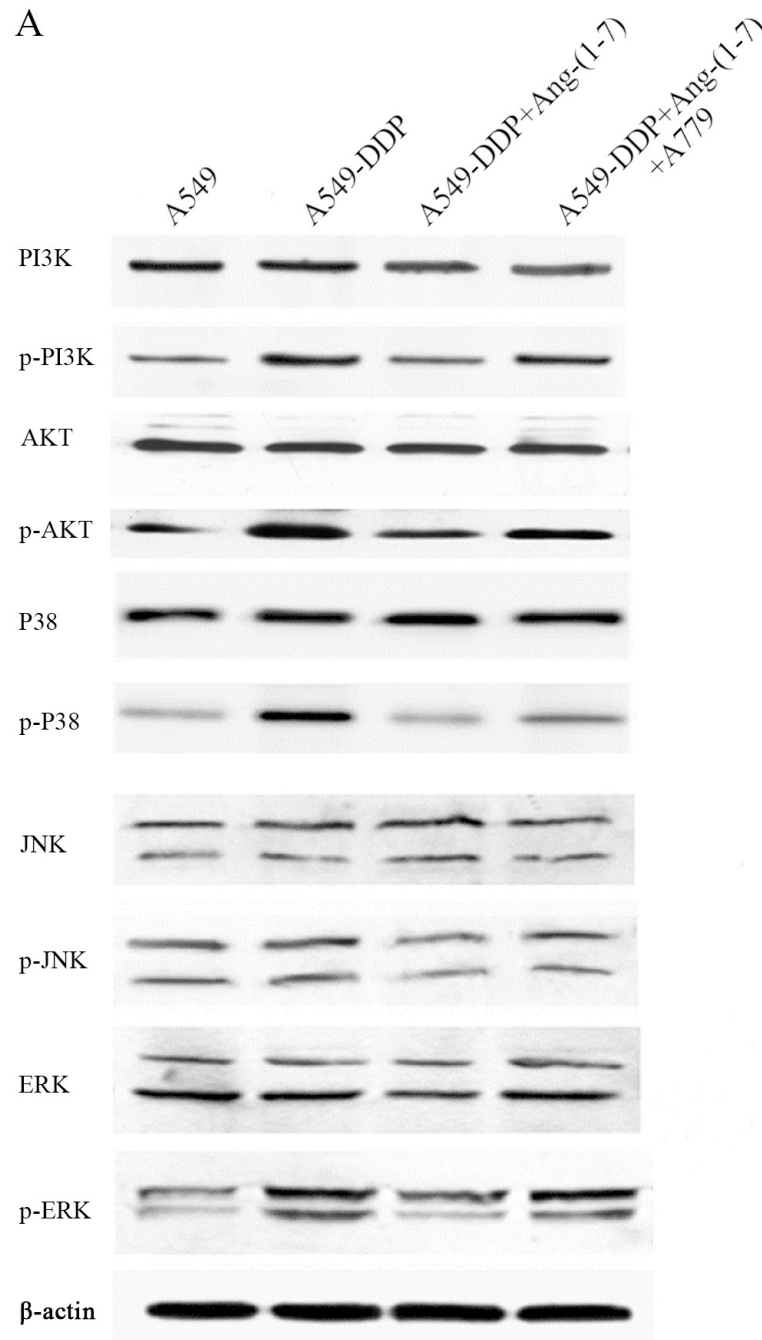

$\mathrm{B}$

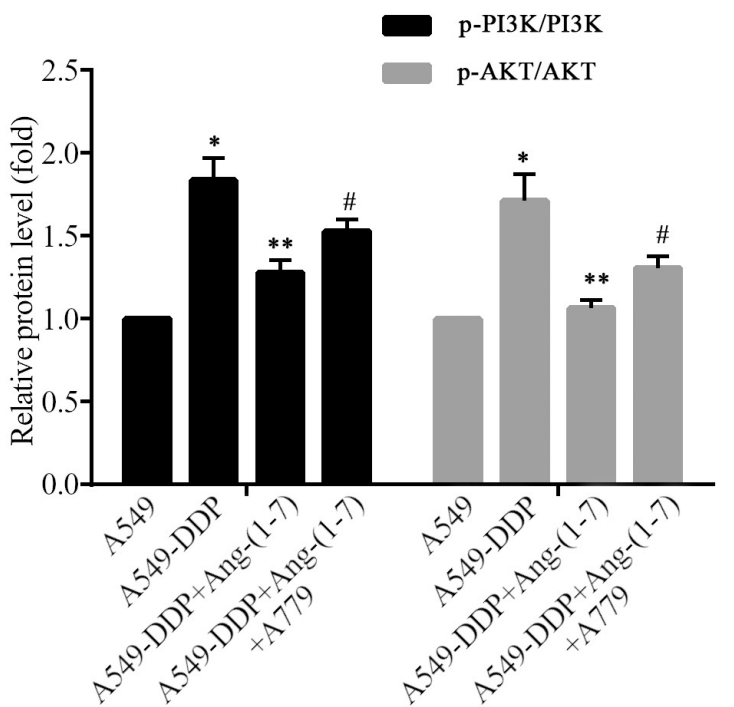

$\mathrm{C}$

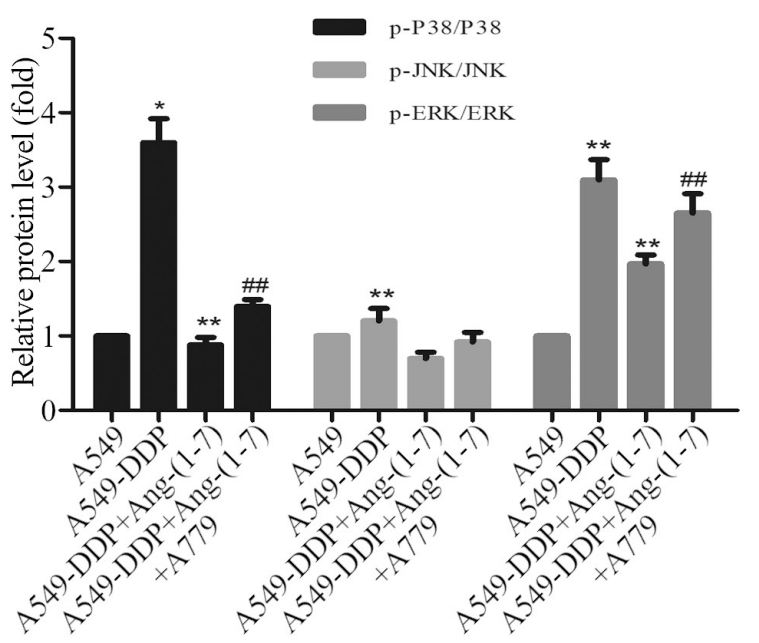

Figure 4. The effect of Ang-(1-7) on the PI3K/Akt and MAPK pathways in A549-DDP cells. A) Western blot detected the protein levels of PI3K/Akt (PI3K, phospho-PI3K, Akt, phospho-Akt) and MAPK signaling (P38, phospho-P38, JNK, phospho-JNK, ERK, phospho-ERK). $\beta$-actin acted as an internal protein. B) Densitometry analyzed p-PI3K and p-Akt levels in A549, A549-DDP, A549-DDP+Ang-(1-7), and A549-DDP+Ang-(1-7)+A779 cells. C) Densitometry analyzed p-P38, p-ERK, and p-JNK levels in A549, A549-DDP, and A549-DDP+Ang-(1-7) and A549-DDP+Ang-(1-7)+A779 groups. 
A

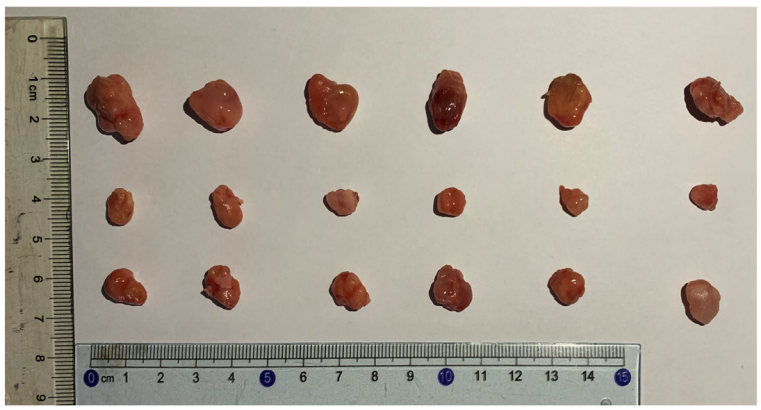

A549-DDP

A549-DDP+Ang-(1-7)

A549-DDP+Ang-(1-7)+A779

B

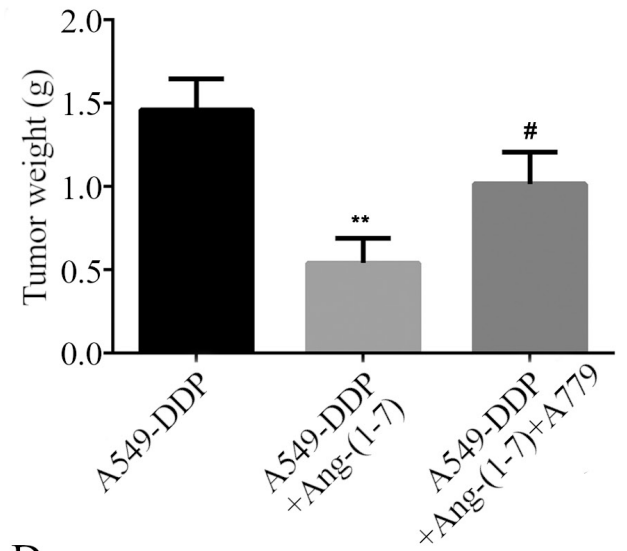

$\mathrm{C}$

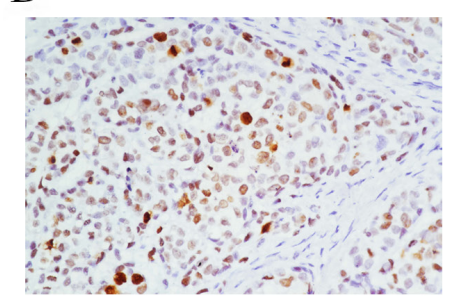

A549-DDP

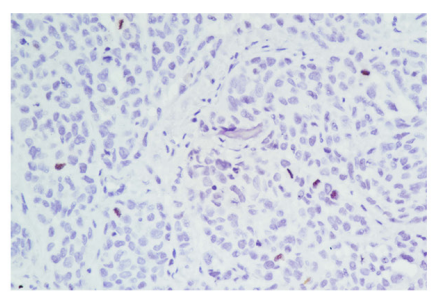

A549-DDP+Ang-(1-7)

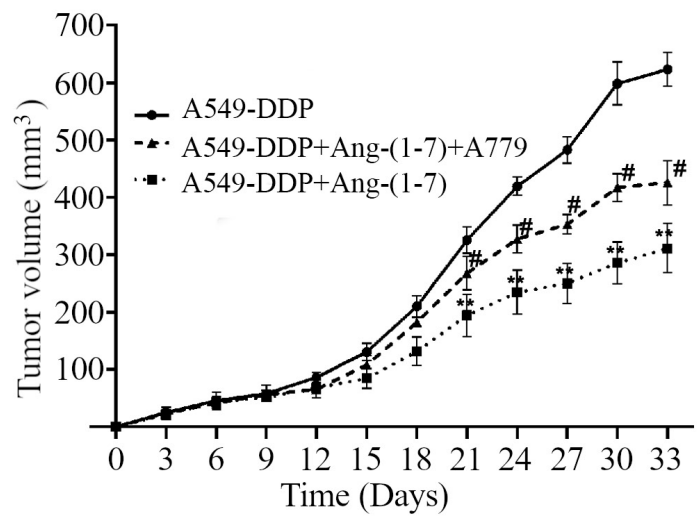

Time (Days)

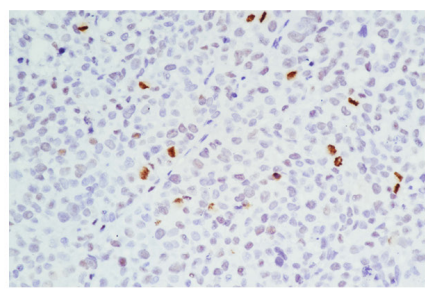

A549-DDP+Ang-(1-7)+A779

Figure 5. Ang-(1-7) inhibits the growth of A549-DDP tumor cells in vivo. A) The tumors of three groups were removed and taken a photo on the 33rd day after inoculation. The impact of the Ang-(1-7) on tumor weight (B) and volume (C). Immunohistochemistry showed that the (D) Ki-67 staining intensity was weaker in the A549-DDP+Ang-(1-7) group than in the A549-DDP group. Data are represented as mean \pm SD of six mice per group. Statistically significant differences are indicated in (A) for tumor weight and (B) for tumor volume ${ }^{\star *}$ p $<0.05$ vs. A549-DDP, ${ }^{*} p<0.05$ vs. A549-DDP+Ang-(1-7).

\section{Discussion}

Here, we demonstrated the impact of Ang-(1-7) on VEGFa production and tumor-associated angiogenesis in NSCLC with the acquired DDP-resistance. At the same time, we analyzed the signaling pathway, which was involved in the VEGFa expression and angiogenesis. Overexpressed Ras or mutation activated Ras could induce abnormal MAPK signaling pathways, which leads to cisplatin resistance [13]. The RAS family includes H-RAS, N-RAS, and K-RAS, which have all been implicated in multiple cancer types. The major signaling pathways involved in RAS activation are the PI3K/ AKT and MAPK signaling pathways [14]. In addition,
HDAC4 and HIF-1a might be two major molecules that mediate p53 and RAS/MAPK signaling to promote cancer cell cisplatin resistance through modulation of autophagy and apoptosis [15]. The ACE2/Ang-(1-7)/Mas axis, which is a new part of Ras, plays a fundamental role in the cancer [16-18]. ACE2 has been shown to associate with cancer prognosis $[19,20]$. A study has shown that Ang-(1-7) has a pleiotropic effect on the treatment of prostate cancer [21]. We previously reported that Ang-(1-7) decreased lung cancer metastasis via inhibition of MMPs [11] as $100 \mathrm{nM}$ Ang-(1-7) significantly reduced A549 invasion and metastasis [11]. In this study, as previously reported, we constructed acquired DDP-resistant NSCLC cell lines [10] and discovered higher 
A

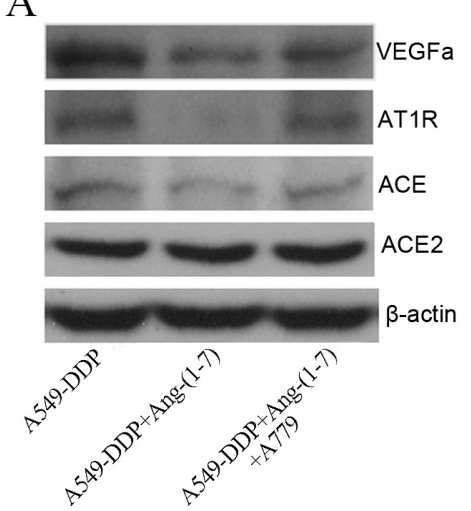

$\mathrm{B}$

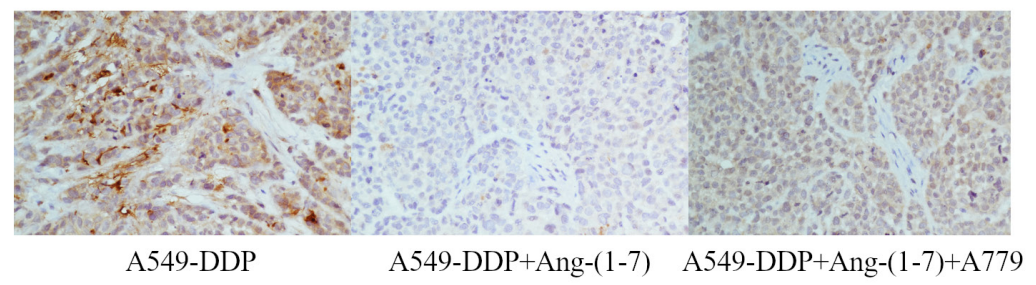

$\mathrm{C}$
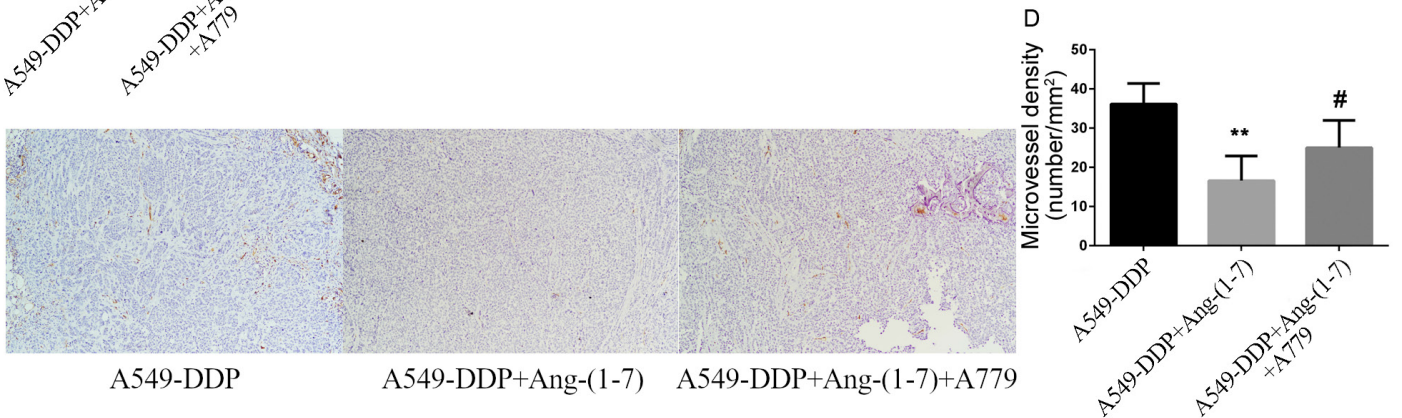

Figure 6. Ang-(1-7) inhibits the tumor growth of A549-DDP in vivo. A) The effects of Ang-(1-7) alone and a combination of Ang-(1-7) and A779 on the production of ACE, VEGFa, ACE2, and AT1R in A549-DDP+Ang-(1-7) cells and A549-DDP cells in vivo. B) Immunohistochemistry analysis analyzed the VEGFa staining intensity, which was weaker in the A549-DDP+Ang-(1-7) group than in the A549-DDP group. C) Immunohistochemical detected the distribution and expression of vascular endothelial cell-specific marker CD34. Representative images for different treatment groups are shown. The positive expression of CD34 is mainly represented as brownish-yellow or brownish granules in vascular endothelial cells. D) Tumor microvessel density (MVD) in different treatment groups. The MVD in A549-DDP+Ang-(1-7) group was significantly lower than that in the A549-DDP group. ${ }^{* *} \mathrm{p}<0.05$ vs. A549-DDP, ${ }^{*} p<0.05$ vs. A549-DDP+Ang-(1-7).

RAS components and VEGF production in A549-DDP and LLC-DDP cells than in parental cells. We then found that Ang-(1-7) inhibited VEGFa production and affected other RAS components' expression in acquired DDP-resistant A549 and LLC cells. Emerging data suggested AT1R plays an important role in acquired platinum resistance-associated neovascularization in bladder cancer [2]. In our previous study, ACE2 was found to inhibit neovascularization associated with acquired platinum resistance in NSCLC [10]. Until now, there is no related research demonstrating the role of Ang-(1-7) and platinum resistance-associated neovascularization in lung cancer.

We then explored pathways that participated in the VEGFa expression and angiogenesis. A recent study showed that Ang-(1-7) inhibits the growth and angiogenesis of hepatocellular carcinoma by the inactivation of P38 phosphorylation [22]. ERK1/2 activity could be inhibited by Ang-(1-7) [23]. ERK1/2 promotes VEGFa expression by stimulating the activator protein-2/Sp-1 complex on the VEGF-A promoter [24]. We also found that Ang-(1-7) suppressed A549 cells metastasis via inhibition of MMP2 and MMP9 [11]. Ang-(1-7) inhibited tumor growth in the xenograft of human nasopharyngeal carcinoma by downregulating the autophagy-mediated PI3K/Akt/mTOR pathway [25].
ACE2/Ang-(1-7)/Mas axis prevents inflammatory response of pancreatic acinar cells by inhibiting p38 MAPK/NF- $\kappa B$ pathway [26]. Consistent with these reports, we observed $\mathrm{PI} 3 \mathrm{~K} / \mathrm{Akt}, \mathrm{P} 38$, and ERK1/2 phosphorylation are involved in the VEGFa production.

In this study, Ang-(1-7) inhibited tumor growth and angiogenesis in the acquired DDP-resistant A549 cells in vivo. Quantification of Ki-67-positive cells showed a decreased proliferation of tumor cells in the A549-DDP+Ang-(1-7) group than in the A549-DDP group. We also demonstrated that the expression of VEGFa and MVD decreased in the A549-DDP+Ang-(1-7) group by IHC analysis. These in vivo and in vitro results are consistent with each other.

In conclusion, these results suggested the Ang-(1-7) could suppress VEGFa expression and tumor-associated angiogenesis in acquired DDP-resistant NSCLC. Our study revealed that targeting Ang-(1-7) may be a new strategy for the treatment of acquired platinum-resistant NSCLC.

Supplementary information is available in the online version of the paper.

Acknowledgments: This study was supported by the National Natural Science Foundation of China (81370130). Yun 
Feng was supported by Shanghai Jiao Tong University Medical cross project (YG2017MS64) and Shanghai Natural Science Foundation (18ZR1424000), Shanghai Municipal Key Clinical Specialty(shslczdzk02202), Shanghai Top-Priority Clinical Key Disciplines Construction Project (2017ZZ02014), and National Innovative Research Team of High-level Local Universities in Shanghai.

\section{References}

[1] DILRUBA S, KALAYDA GV. Platinum-based drugs: past, present and future. Cancer Chemother Pharmacol 2016; 77: 1103-1124. https://doi.org/10.1007/s00280-016-2976-Z

[2] TANAKA N, MIYAJIMA A, KOSAKA T, MIYAZAKI Y, SHIROTAKE $S$ et al. Acquired platinum resistance enhances tumour angiogenesis through angiotensin II type 1 receptor in bladder cancer. Br J Cancer 2011; 105: 1331-1337. https:// doi.org/10.1038/bjc.2011.399

[3] PYASKOVSKAYA ON, DASYUKEVICH OI, KOLESNIK DL, GARMANCHOUK LV, TODOR IN et al. Changes in VEGF level and tumor growth characteristics during lewis lung carcinoma progression towards cis-DDP resistance. Exp Oncol 2007; 29: 197-202.

[4] FERREIRA AJ, SANTOS RAS. Cardiovascular actions of angiotensin-(1-7). Braz J Med Biol Res 2005; 38: 499-507. https://doi.org/10.1590/s0100-879x2005000400003

[5] FERRARIO CM, VARAGIC J. The ANG-(1-7)/ACE2/mas axis in the regulation of nephron function. Am J Physiol Renal Physiol 2010; 298: F1297-305. https://doi.org/10.1152/ ajprenal.00110.2010

[6] ZHAO Y, WANG H, LI X, CAO M, LU H et al. Ang II-AT1R increases cell migration through PI3K/AKT and NF-kappaB pathways in breast cancer. J Cell Physiol 2014; 229: 18551862. https://doi.org/10.1002/jcp.24639

[7] IMAI N, HASHIMOTO T, KIHARA M, YOSHIDA SI, KAWANA I et al. Roles for host and tumor angiotensin II type 1 receptor in tumor growth and tumor-associated angiogenesis. Lab Invest 2007; 87: 189-198. https://doi. org/10.1038/labinvest.3700504

[8] FENG Y, WAN H, LIU J, ZHANG R, MA Q et al. The angiotensin-converting enzyme 2 in tumor growth and tumorassociated angiogenesis in non-small cell lung cancer. Oncol Rep 2010; 23: 941-948. https://doi.org/10.3892/or_00000718

[9] FENG Y, NI L, WAN H, FAN L, FEI X et al. Overexpression of ACE2 produces antitumor effects via inhibition of angiogenesis and tumor cell invasion in vivo and in vitro. Oncol Rep 2011; 26: 1157-1164. https://doi.org/10.3892/ or.2011.1394

[10] CHENG Q, ZHOU L, ZHOU J, WAN H, LI Q et al. ACE2 overexpression inhibits acquired platinum resistance-induced tumor angiogenesis in NSCLC. Oncol Rep 2016; 36: 1403-1410. https://doi.org/10.3892/or.2016.4967

[11] NI L, FENG Y, WAN H, MA Q, FAN L et al. Angiotensin-(1-7) inhibits the migration and invasion of A549 human lung adenocarcinoma cells through inactivation of the PI3K/Akt and MAPK signaling pathways. Oncol Rep 2012; 27: 783-790. https://doi.org/10.3892/or.2011.1554
[12] SOTO-PANTOJA DR, MENON J, GALLAGHER PE, TALLANT EA. Angiotensin-(1-7) inhibits tumor angiogenesis in human lung cancer xenografts with a reduction in vascular endothelial growth factor. Mol Cancer Ther 2009; 8: 1676 1683. https://doi.org/10.1158/1535-7163.MCT-09-0161

[13] KENNEDY AL, MORTON JP, MANOHARAN I, NELSON DM, JAMIESON NB et al. Activation of the PIK3CA/AKT pathway suppresses senescence induced by an activated RAS oncogene to promote tumorigenesis. Mol Cell 2011; 42: 3649. https://doi.org/10.1016/j.molcel.2011.02.020

[14] RITT DA, ABREU-BLANCO MT, BINDU L, DURRANT DE, ZHOU $\mathrm{M}$ et al. Inhibition of Ras/Raf/MEK/ERK Pathway Signaling by a Stress-Induced Phospho-Regulatory Circuit. Mol Cell 2016; 64: 875-887. https://doi.org/10.1016/j. molcel.2016.10.029

[15] ZHANG X, QI Z, YIN H, YANG G. Interaction between p53 and Ras signaling controls cisplatin resistance via HDAC4and HIF-1 $\alpha$-mediated regulation of apoptosis and autophagy. Theranostics 2019; 9: 1096-1114. https://doi.org/10.7150/ thno. 29673

[16] XU J, FAN J, WU F, HUANG Q, GUO M et al. The ACE2/ Angiotensin-(1-7)/Mas Receptor Axis: Pleiotropic Roles in Cancer. Front Physiol 2017; 8: 276. https://doi.org/10.3389/ fphys.2017.00276

[17] ENDER SA, DALLMER A, LASSIG F, LENDECKEL U, WOLKE C. Expression and function of the ACE2/angiotensin(1-7)/Mas axis in osteosarcoma cell lines U-2 OS and MNNG-HOS. Mol Med Rep 2014; 10: 804-810. https://doi. org/10.3892/mmr.2014.2266

[18] FAN L, FENG Y, WAN HY, NI L, QIAN YR et al. Hypoxia induces dysregulation of local renin-angiotensin system in mouse Lewis lung carcinoma cells. Genet Mol Res 2014; 13: 10562-10573. https://doi.org/10.4238/2014

[19] LI J, YANG ZL, REN X, ZOU Q, YUAN Y et al. ACE2 and FZD1 are prognosis markers in squamous cell/adenosquamous carcinoma and adenocarcinoma of gallbladder. J Mol Histol 2014; 45: 47-57. https://doi.org/10.1007/s10735-0139528-1

[20] YE G, QIN Y, LU X, XU X, XU S et al. The association of renin-angiotensin system genes with the progression of hepatocellular carcinoma. Biochem Biophys Res Commun 2015; 459: 18-23. https://doi.org/10.1016/j.bbrc.2015.02.030

[21] KRISHNAN B, TORTI FM, GALLAGHER PE, TALLANT EA. Angiotensin-(1-7) reduces proliferation and angiogenesis of human prostate cancer xenografts with a decrease in angiogenic factors and an increase in sFlt-1. Prostate 2013; 73: 60-70. https://doi.org/10.1002/pros.22540

[22] LIU Y, LI B, WANG X, LI G, SHANG R et al. Angiotensin-(1-7) Suppresses Hepatocellular Carcinoma Growth and Angiogenesis via Complex Interactions of Angiotensin II Type 1 Receptor, Angiotensin II Type 2 Receptor and Mas Receptor. Mol Med 2015; 21: 626-636. https://doi. org/10.2119/molmed.2015.00022

[23] GALLAGHER PE, TALLANT EA. Inhibition of human lung cancer cell growth by angiotensin-(1-7). Carcinogenesis 2004; 25: 2045-2052. https://doi.org/10.1093/carcin/bgh236 
[24] BERRA E, MILANINI J, RICHARD DE, LE GALL M, VINALS F et al. Signaling Angiogenesis via p42/p44 MAP Kinase and Hypoxia. Biochem Pharmacol 2000; 60: 1171-1178. https://doi.org/10.1016/s0006-2952(00)00423-8

[25] LIN YT, WANG HC, CHUANG HC, HSU YC, YANG MY et al. Pre-treatment with angiotensin-(1-7) inhibits tumor growth via autophagy by downregulating PI3K/Akt/ mTOR signaling in human nasopharyngeal carcinoma xenografts. J Mol Med (Berl) 2018; 96: 1407-1418. https://doi. org/10.1007/s00109-018-1704-Z
[26] YU X, CUI L, HOU F, LIU X, WANG Y et al. Angiotensinconverting enzyme 2-angiotensin (1-7)-Mas axis prevents pancreatic acinar cell inflammatory response via inhibition of the p38 mitogen-activated protein kinase/nuclear factorkappaB pathway. Int J Mol Med 2018; 41: 409-420. https:// doi.org/10.3892/ijmm.2017.3252 
https://doi.org/10.4149/neo_2021_201213N1347

\section{The role of angiotensin-(1-7) on acquired platinum resistance-induced angiogenesis in non-small cell lung cancer in vitro and in vivo}

Yan-Lai GENG ${ }^{1,2,3, *}$, Yong-Jie DING ${ }^{1,2, *}$, Lei NI ${ }^{1,2}$, Kan-Di XU ${ }^{1,2}$, Van-Minh LE $E^{4}$, Ri JI ${ }^{5, *}$, Yun FENG ${ }^{1,2, *}$

Supplementary Information

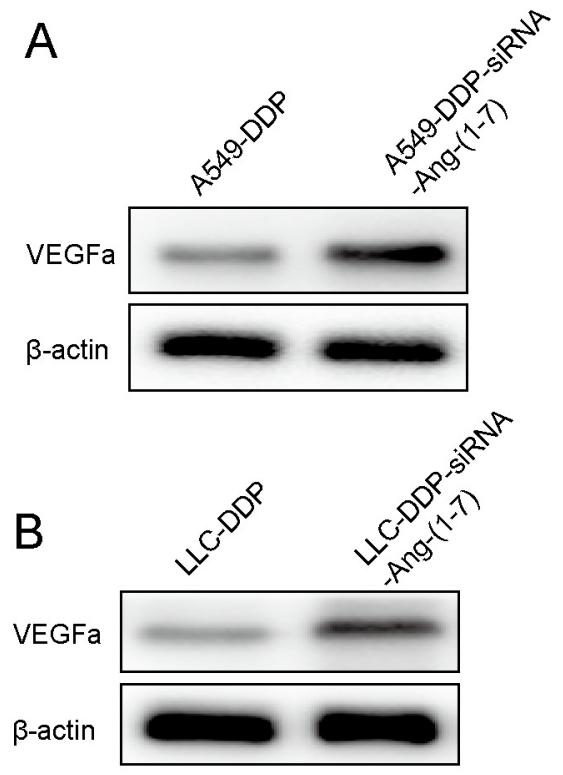

C

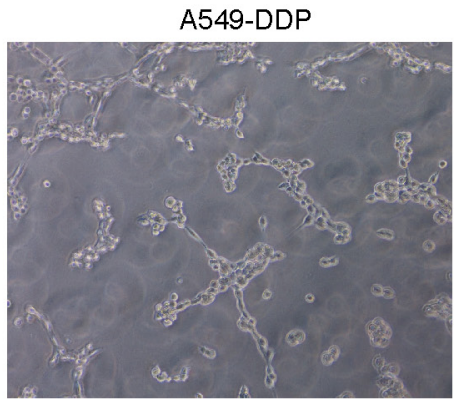

A549-DDP-siRNA-Ang-(1-7)

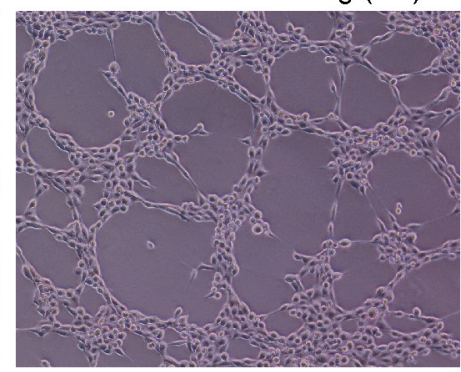

$\mathrm{D}$

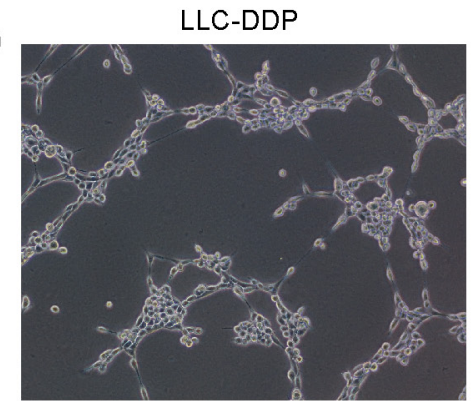

LLC-DDP-siRNA-Ang-(1-7)

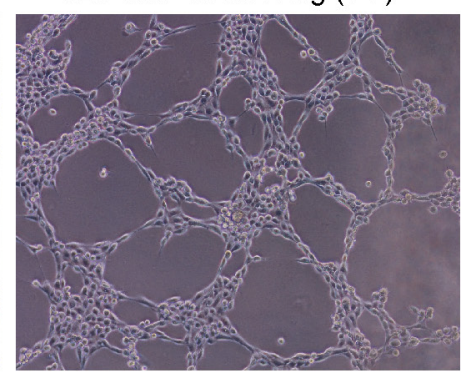

Supplementary Figure S1. The effects of si-Ang-(1-7) on VEGFa productions and tube formations in the A549/LLC-DDP cells. The effects of siAng-(1-7) on the production of VEGFa in the A549-DDP-siRNA-Ang-(1-7) cells and A549-DDP cells (A) and the LLC-DDP-siRNA-Ang-(1-7) cells and LLC-DDP cells (B). Representative images of tube formation in the A549-DDP and A549-DDP-siRNA-Ang-(1-7) (C) and the LLC-DDP cells and LLC-DDP-siRNA-Ang-(1-7) cells (D) cultured on Matrigel for $24 \mathrm{~h}$. 
A

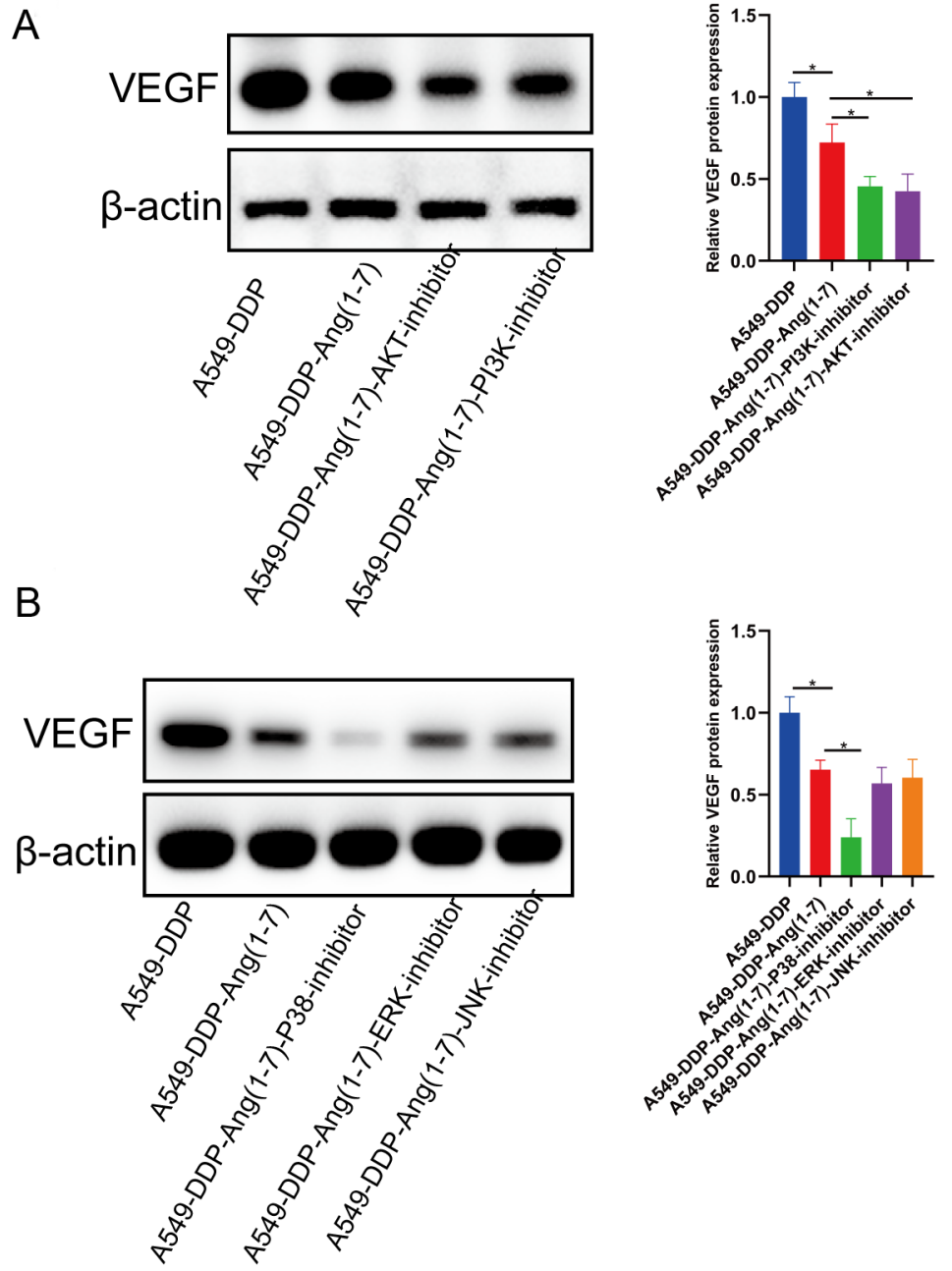

Supplementary Figure S2. The impact of inhibiting PI3K/Akt and MAPK signaling on the expression of VEGF. Western blot detected A) the impact of PI3K/Akt inhibitors and B) MAPK inhibitors on the protein levels of VEGF. 